\title{
Luminosity Measurements at Hadron Colliders
}

\author{
Vaia Papadimitriou* \\ Fermi National Accelerator Laboratory, P.O. Box 500, Batavia, Illinois, 60510, U.S.A.
}

Elsevier use only: Received date here; revised date here; accepted date here

\begin{abstract}
In this paper we discuss luminosity measurements at Tevatron and HERA as well as plans for luminosity measurements at LHC. We discuss luminosity measurements using the luminosity detectors of the experiments as well as measurements by the machine. We address uncertainties of the measurements, challenges and lessons learned.

(C) 2008 Elsevier B.V. All rights reserved
\end{abstract}

PACS: 01.30.Cc; 29.40.Ka; 29.40.Mc; 20.40.Vj; 29.40.Cs; 29.40.Wk

Keywords: Luminosity; hadron; Tevatron; HERA; LHC

\section{Introduction}

Luminosity measurements are an absolutely necessary component of any experimental beam colliding program since they provide the frequency of the interactions and the needed normalization for the physics process under study (Standard Model and beyond the Standard Model physics processes, new physics, etc.). Luminosity measurements also allow for the monitoring of the performance of the accelerator and for the implementation of beam parameter adjustments as needed for optimized performance.

We will discuss below luminosity measurements by the CDF and D0 experiments on the Tevatron side and by the $\mathrm{H} 1$ and ZEUS experiments on the HERA side. We will review the techniques used and discuss the uncertainties of the measurements. We will also discuss the challenges that were faced and the lessons learned. On the LHC side we will discuss the plans for luminosity measurements by the machine as well as by the experiments. In this case we will focus on the high luminosity regions of ATLAS and CMS. We will discuss again techniques, expected uncertainties and challenges to be faced.

\section{Luminosity measurements at the Tevatron}

The Tevatron, a proton-antiproton collider, has delivered
$110 \mathrm{pb}^{-1}$ per experiment to the CDF and D0 experiments in Run I (1992 - 1996) at a center of mass energy of $1.8 \mathrm{TeV}$ and with a $3.5 \mu$ s spacing between collisions. It has in addition delivered $3.85 \mathrm{fb}^{-1}$ per experiment between July 2001 and March 2008 (Run II) at a center of mass energy of $1.96 \mathrm{TeV}$ and with a 396 ns spacing between collisions. Thirty-six proton bunches are colliding with thirty six antiproton bunches in Run II, with a typical number of protons per bunch, $\sim 2.5 \mathrm{x}$ $10^{11}$ and a typical number of antiprotons per bunch $\sim 7 \times 10^{10}$. On March 17, 2008 there was an initial luminosity record set of $3.15 \times 10^{32} \mathrm{~cm}^{-2} \mathrm{~s}^{-1}$. Between March 24 and March 30, 2008 the accelerator complex delivered a record of $48 \mathrm{pb}^{-1}$ for a single week. The Tevatron is expected to deliver 5.8-6.7 $\mathrm{fb}^{-1}$ per experiment by the end of fiscal year 2009 and, if it runs longer, $7.3-8.8 \mathrm{fb}^{-1}$ by the end of fiscal year 2010 .

Absolute luminosity measurements by the machine based on measurements of beam parameters like emittances, intensities, beam lattice etc. have uncertainties of the order of 15-20\%. The Tevatron luminosity measurements are basically based on the real time, relative luminosity measurements performed by the CDF and D0 experiments which are then normalized to a relatively well known and copious process, in this case the inclusive, inelastic proton-antiproton cross

* Corresponding author. Tel.: +1-630-840-8207; fax: +1-630-840-4552.

E-mail address: vaia@fnal.gov. 
section. The instantaneous luminosity $\mathcal{L}$ is being estimated by using Equation 1, where $\mu$ is the average number of interactions per beam crossing, $\mathrm{f}_{\mathrm{BC}}$ is the frequency of bunch crossings and $\sigma_{\text {in }}$ is the inelastic cross section. The average number of interactions can be estimated either by measuring the probability of zero interactions $\left(P_{0}(\mu)=e^{-\mu}\right.$ for a detector of $100 \%$ acceptance) or directly, by counting particles or hits or time clusters in the detector. The CDF and D0 collaborations have agreed to use a common proton-antiproton inelastic cross section for luminosity normalization in Run II. This common cross section has been derived [1] on the basis of averaging the inelastic cross sections measured by the Fermilab CDF and E811 experiments at $1.8 \mathrm{TeV}$ and extrapolating the cross section at $1.96 \mathrm{TeV}$.

$$
\mu \cdot f_{B C}=\sigma_{\text {in }} \cdot \mathcal{L} \quad(1)
$$

In addition, one can cross calibrate the luminosity measurements with rarer, cleaner and better understood processes like the decay $W \rightarrow l \cdot v$.

Both experiments have used scintillating counters to measure the luminosity during Run I. For Run II, where the instantaneous luminosity is substantially higher, CDF opted for a Cherenkov counter system while D0 for a scintillating counter system of better granularity than Run I.

The CDF Cherenkov counter system [2] consists of 48 counters per side arranged in 3 layers with 16 counters each, covering the pseudorapidity region $3.7 \leq|\eta| \leq 4.7$. The counters are filled with isobutene and are being read by Hamamatsu R5800QCC Photomultipliers (PMTs) with a quartz window. The Cherenkov counter system allows for good separation between primaries and secondaries, good amplitude resolution $(\sim 18 \%$ from photostatistics, light collection and PMT collection), good timing resolution and in addition it is radiation hard. Full simulation with PYTHIA agrees well with the data for the amplitude distribution in the Cherenkov counters. In Fig. 1 is displayed the amplitude distribution in the data for one Cherenkov counter after an isolation requirement of less than 20 photoelectrons in the surrounding counters. The Single Particle Peak (SPP) is clear. Fig. 2 shows how the average number of particles (total amplitude over the amplitude of the SPP) or hits (counters with amplitude above a certain threshold) varies as a function of the average number of proton-antiproton interactions and compares the data with the Monte Carlo simulation. The data and the simulation compare very well. At the highest luminosities the particle counting algorithm is more linear. As a reference, note that $\mu$ approximately equal to 6 corresponds to $\mathcal{L}$ approximately equal to $2 \times 10^{32} \mathrm{~cm}^{-2} \mathrm{~s}^{-1}$. The CDF luminosity measurement is based as a default on measuring the probability of zero interactions and uses measuring hits and particles as a cross check. CDF has evaluated that the luminosity measurement using the probability of zero interactions is reliable up to about $3.6 \times 10^{32} \mathrm{~cm}^{-2} \mathrm{~s}^{-1}$. The current CDF luminosity measurement uncertainty is 5.8\%. The leading contribution is from normalizing to the protonantiproton inelastic cross section (4\%). The next two most important contributions are due to simulating the material in the detector (3\%) and the relative contribution from non-

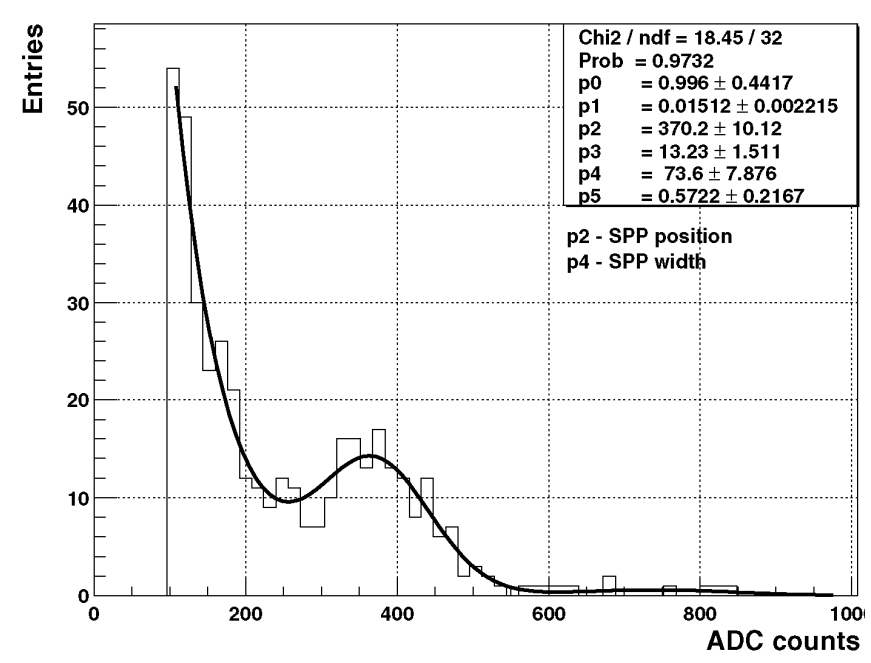

Fig. 1. Amplitude distribution for a single Cherenkov counter at CDF. The solid line represents a fit to the data.

diffractive and diffractive processes in the Monte Carlo generator (2\%). CDF is cross-checking their absolute luminosity measurements by comparing with the inclusive $\mathrm{W}$ and $\mathrm{Z}$ boson cross section measurements and the comparison is very satisfactory. The yield of $J / \psi$ 's and $W$ 's through the $J / \psi \rightarrow \mu \mu$ and $W \rightarrow l v$ decays as a function of instantaneous luminosity serves as an additional check of the stability of the luminosity measurements. The aging rate of the PMTs is $\sim 35 \%$ per $\mathrm{fb}^{-1}$ and is being addressed by High Voltage and PMT gain adjustments or with replacements as needed.

The D0 Run II luminosity system [3] consists of two forward scintillator arrays covering the pseudorapidity region $2.7 \leq|\eta| \leq 4.4$. There are 24 wedges per array, each read out with a fine mesh PMT. Inelastic collisions are being identified by using the coincidence of in-time hits in the two arrays. Since October 2005 the luminosity readout electronics changed from NIM to custom VME [4]. The D0 luminosity measurement is based on measuring the probability of zero interactions. The current D0 luminosity measurement uncertainty is $6.1 \%$. The leading contribution is from normalizing to the proton-antiproton inelastic cross section (4\%). The next two most important contributions are due to the determination of the non-diffractive fraction ( 4\%) and the long term stability ( 2.8\%). Fig. 3 shows a data-Monte Carlo simulation comparison of counter multiplicity (above a threshold) assuming the final, non-diffractive fraction of $0.687 \pm 0.044$. D0 is using the yield of forward muons as a function of time and instantaneous luminosity as an additional check of the stability of the luminosity measurements (within $\sim 1 \%$ during the past couple of years). The radiation damage to the scintillator is being addressed by annealing and replacement as needed.

The CDF/D0 ratio of instantaneous luminosities is being checked continuously and is being compared with the expected ratio on the basis of beam parameters. The goal is to keep this ratio within a couple of percent around 1 . Significantly larger deviations observed a few times so far 
have led to thorough investigation on both the machine and experiment sides and resulted to either machine parameter adjustments or to improvements in the techniques used by the experiments to measure the luminosity [4].

Some of the lessons learned so far from the luminosity measurements at the Tevatron are that: the method of counting zero interactions works well for the current Tevatron luminosities; fine granularity detectors are needed for high instantaneous luminosities (Run I vs Run II); in situ calibration of the detector is very important; detector stability is crucial; a good simulation of the processes involved and of the luminosity detector itself is needed as early as possible; a good knowledge of the physics cross section the measurement relies upon is necessary; careful monitoring of gas purity when having a gas detector is a must; minimizing - eliminating if possible - the dead time of the luminosity system is critical; watchfulness is needed for aging due to large total luminosity and readiness to replace consumables; continuous cross checking between the machine expectations and the measured luminosities by the experiments, as well as between the experiments themselves, is very valuable.

\section{Luminosity measurements at HERA}

HERA, a proton-electron (positron) collider, has delivered $779.9 \mathrm{pb}^{-1}$ per experiment to the $\mathrm{H} 1$ and ZEUS experiments between May 1993 and June 2007. In the most recent period of running, HERA II (2002-2007), the proton beam energy was $920 \mathrm{GeV}$ and the electron (positron) beam energy 27.5 $\mathrm{GeV}$. The spacing between collisions was 96 ns and the initial luminosity record set was $5 \times 10^{31} \mathrm{~cm}^{-2} \mathrm{~s}^{-1}$, to be compared with $1.8 \times 10^{31} \mathrm{~cm}^{-2} \mathrm{~s}^{-1}$ in HERA I. Due to various demands on the operation of the machine, occasionally compromises had to be reached between higher luminosity and better background conditions or higher luminosity and higher polarization.

Two methods have been used to measure luminosity at HERA: a) The H1 and ZEUS experiments used their own luminosity systems counting the rate of bremsstrahlung Bethe-Heitler events with (2-5) \% uncertainty online and (1-3) \% uncertainty offline. b) The machine every few years performed beam scans, calculated from them emittances and estimated the expected luminosity folding-in beta functions and assuming perfect beam spot overlaps at the interaction points (IPs). This measurement of the luminosity had an uncertainty of approximately $10 \%$ where the main uncertainty was from the beta function at the IP. There was a (5-10) \% luminosity difference between the two IPs.

The Bethe-Heitler process, $e p \rightarrow e^{\prime} p \gamma$, has an accurately calculable cross section as well as sufficient rate for real time monitoring [5]. The main background comes from beam gas scattering and is calculated and subtracted by using electron pilot bunches.

The main two challenges for luminosity measurements at HERA II were the increased synchrotron radiation level (higher total power and harder spectrum) as well as the increased BetheHeitler event rate due to the higher luminosity and hence pile-up. These required fast and radiation hard detectors and electronics.

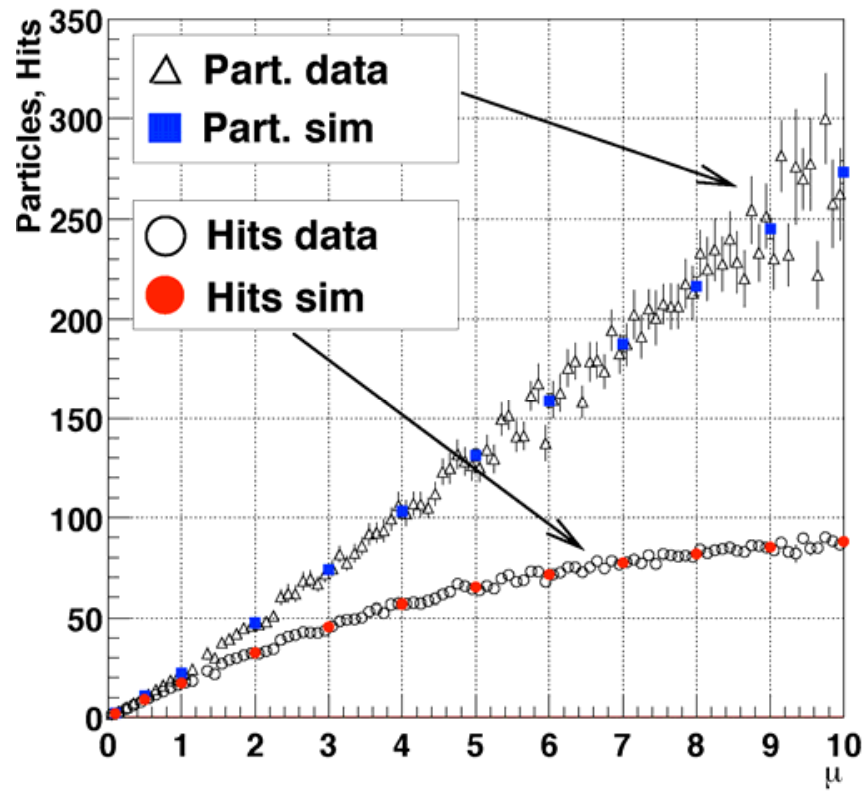

Fig. 2. Data vs Monte Carlo simulation comparison of the average number of particles or hits vs the average number of proton-antiproton interactions at CDF.

The H1 luminosity detector for HERA II [6] consisted of a photon detector (tungsten/quartz-fibre calorimeter) at 104 meters from the IP and an electron tagger (a lead "spaghetti" calorimeter with a high volume fraction of scintillating fibres) at 6 meters from the IP for the detection of scattered electrons. A synchrotron radiation filter (two radiation lengths of Beryllium) was also part of the photon detection system.

ZEUS used two independent luminosity monitors with different systematics $[7,8]$. Their system consisted of a radiation hard lead-scintillator calorimeter positioned 107 meters from the IP to detect the photons, an electron tagger calorimeter positioned 6 meters from the IP for the detection of scattered electrons, and an electron-positron pair spectrometer. As far as the photon detection is concerned, an older system [7] was modified by adding active filters in order to suppress the increased synchrotron radiation background of HERA II. The electron-positron magnetic spectrometer [8], exploited the fact that about $9 \%$ of Bethe-Heitler photons were converting in the photon exit window which was located in the end of the vacuum chamber. The resulting electrons and positrons were detected by a tungsten-scintillator calorimeter. In principle there was no risk of radiation damage to this calorimeter since it was away from the synchrotron radiation plane and the bremsstrahlung photon beam. When back-scattered synchrotron radiation started becoming a problem, improved shielding and frequent calibrations addressed the issue.

The H1 luminosity measurement uncertainty for HERA I was $1.5 \%$ [9]. The current uncertainty for HERA II is (2.5-3.0) $\%$ and the goal is to reach a $2 \%$ uncertainty. In contrast with the Tevatron experiments, normalization to the calculated Bethe-Heitler cross section contributes only $0.5 \%$ to the above uncertainty (the same is true for the ZEUS measurement as well). The leading contribution is from the calculation of the acceptance (1-2) \%. 
The ZEUS luminosity measurement uncertainty for HERA I was $1.05 \%$ [7]. The current uncertainty for HERA II is $2.6 \%$ and the goal is to reach eventually a $2 \%$ uncertainty. The leading contribution to the uncertainty is from understanding the photon conversion rate at the exit window (2\%).

Some of the lessons learned from the luminosity measurements at HERA are that: for detectors close to beams and/or exposed to harsh conditions, radiation levels need to be estimated thoroughly; one should not count on the calculated optics, on perfect alignment and ideal running conditions since the real machine can be more difficult than anticipated; one has to be ready for surprises like the unexpected proton satellites which caused running at a compromised acceptance instead of the ideal one; using more than one method for luminosity determination is essential for reducing the systematics; one never has too many slow control and cross calibration systems, especially in harsh environments that can not be reproduced unless one has the real beam; the pile-up was expected and well handled. In the end, radiation resistance was achieved using efficient shielding and the background underestimation was remedied by this shielding and dynamic pedestal subtraction.

\section{Luminosity measurements at LHC}

LHC is a proton-proton collider with expected center of mass energy of $14 \mathrm{TeV}$ and with a $25 \mathrm{~ns}$ spacing between collisions. The machine's expected design luminosity is $10^{34}$ $\mathrm{cm}^{-2} \mathrm{~s}^{-1}$. It is also anticipated that there will be a low luminosity running period in the beginning, with luminosities of the order of $10^{32} \mathrm{~cm}^{-2} \mathrm{~s}^{-1}$. The high luminosity will be achieved by filling the LHC with 2808 bunches of $10^{11}$ protons per bunch. Operation of the LHC is expected to start in the summer of 2008.

Four large-scale experiments will perform high energy particle physics research in the LHC. ATLAS and CMS are general purpose experiments located at the high luminosity IPs 1 and 5. LHCb (study of b-quarks and CP violation) and ALICE (a heavy-ion experiment for quark-gluon plasma studies) are located respectively at the lower luminosity IPs 8 and 2.

The plan for the experiments and the machine is to measure the absolute luminosity with a precise method at optimal conditions. In addition, provide relative (real time) luminosity measurements using dedicated luminosity monitors provided by either the experiments or the machine. Then the luminosity monitors will be calibrated with the absolute luminosity measurement. The uncertainty in the beginning is expected to be of the order of $20 \%$ while the aim is to reduce it to below $5 \%$ after some years of experience.

The machine plans to measure in real time the rate of neutral particles like neutrons and photons, generated either directly or from the decay of unstable particles, using collision rate monitors [10]. Due to the very different luminosity levels at the four IPs there are two different types of collision rate monitors being developed; ionization chambers for the high

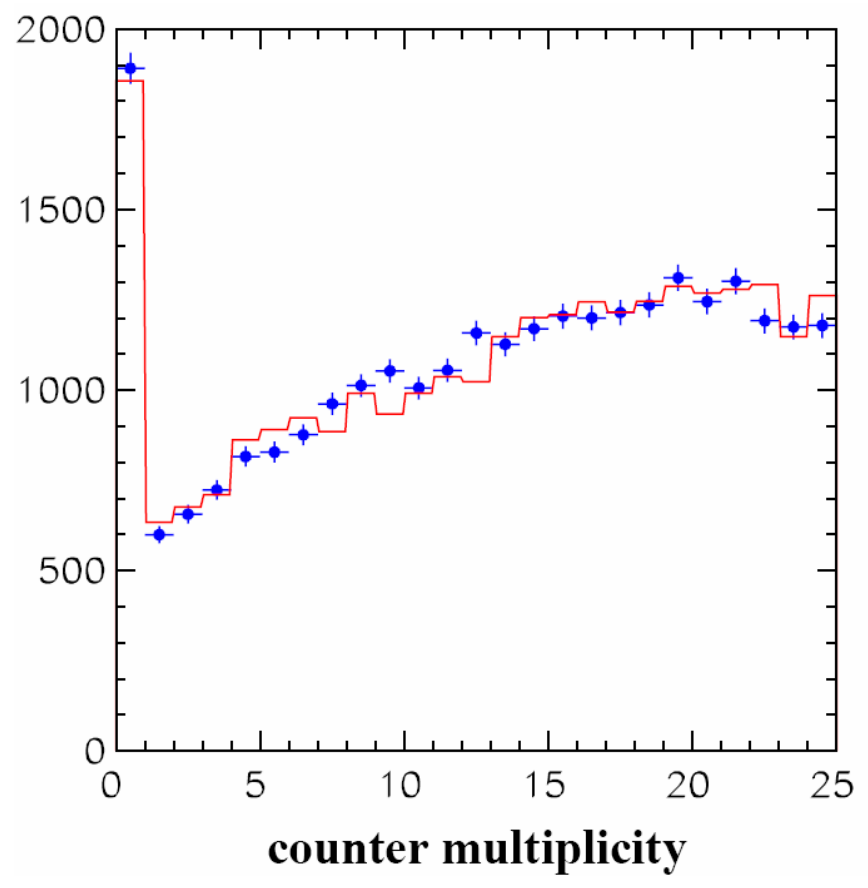

Fig. 3. Data vs Monte Carlo simulation comparison of the multiplicity of the luminosity counters at D0 using the final non-diffractive fraction. The points represent the data and the solid line the Monte Carlo. The plot corresponds to an instantaneous luminosity of $1.3 \times 10^{31} \mathrm{~cm}^{-2} \mathrm{~s}^{-1}$.

luminosity regions and solid state polycrystalline Cadmium Telluride detectors for the lower luminosity regions. Both types of detectors are to be installed inside the TANs, absorbers made of copper several meters long and located just in front of the D2 magnets at the IPs.

Absolute luminosity measurements by the machine using beam parameters are expected to have initial uncertainties of the order of (20-30) \%. The machine plans to perform as well special calibration runs at low luminosity and therefore improve the determination of the overlap integral of the two beams at the interaction regions [11]. The overlap integral in this case will be measured by displacing the beams with respect to each other separately in the horizontal and vertical directions (Van der Meer scans). An uncertainty of less than 5\% may be possible using this method but then this absolute calibration will have to be transferred to high luminosities.

The goals of ATLAS and CMS are to measure the absolute luminosity considering two major approaches [12, 13]: a) using the rate of well calculable processes like the production of $\mathrm{W}$ and $\mathrm{Z}$ bosons or dilepton production via two photon exchange and b) using the optical theorem from a simultaneous measurement of the total interaction rate and the rate of forward elastic scattering. Measuring the luminosity using Coulomb scattering is being discussed as well. Approach a) can be used at all levels of instantaneous luminosities while approach b) is meant to be used at lower instantaneous luminosities. The measurement from approach b) will be then extrapolated to higher luminosities. For approach a), the advantage is that $\mathrm{W}$ and $\mathrm{Z}$ boson production has high rate; on the other hand it has some theoretical limitations, currently uncertainties of the order of (5-10) \%. 
The dilepton production via two photon exchange has very small theoretical uncertainties $(\sim 1 \%)$ but the rate is low and there will be challenges in the calculation of the efficiency [14]. One of the main challenges of approach b) lies in the detection of the very forward elastically-scattered protons. The uncertainty in the luminosity measurements using this approach can be of the order of a few \% but the measurements require special runs and the beam conditions during those runs can be demanding. There are plans as well to use a combination of techniques to measure the absolute luminosity, e.g. using the total cross section as measured by other experiments or combining the absolute luminosity measurement of the machine with the optical theorem. As far as implementing approach b) is concerned, ATLAS is preparing to measure elastic scattering by using scintillatingfibre trackers housed inside sets of Roman Pots (ALFA system) located at 240 meters at each side of IP 1 [15]; CMS is preparing to use the measurement of the total proton-proton cross section by the TOTEM experiment [16] which is installed at IP 5. TOTEM is dedicated to the measurement (at low luminosity) of the total proton-proton cross section, elastic scattering and diffractive processes at the LHC. It will measure elastic scattering using three sets of Roman Pots at each side of IP 5 and in addition it will measure inelastic interactions in the forward region by using two tracking telescopes.

The ATLAS experiment is preparing to make real time luminosity measurements by using a Cherenkov counter system (LUCID) very similar to the one used by the CDF experiment and located at a distance of 17 meters from the IP [15]. The CMS experiment is preparing to make real time luminosity measurements by using their Hadronic Forward (HF) Calorimeter consisting of radiation hard quartz fibres embedded into steel absorbers. The plan is to use the HF tower occupancy to count the number of empty bunch crossings to infer $\mu$ (see equation 1). An alternative method is expected to exploit the linear relationship between the average transverse energy per tower deposited in the HF and the number of interactions per bunch crossing. In addition, CMS is discussing the possibility to use arrays of small angle pixel luminosity telescopes (PLT) each consisting of three planes of diamond pixel sensors. The PLT systematics are expected to be complimentary to the ones of the HF calorimeter.

The plan of the LHCb experiment is to measure the luminosity by using beam-gas interactions reconstructed in the LHCb vertex detector [17]. Studies are also in progress in order to measure the luminosity by using the rate of pairs of muons either from the decay of the $\mathrm{Z}$ bosons or from twophoton processes.

The ALICE experiment is planning to measure real time luminosity by using a combination of two rings of plastic scintillators (V0), two arrays of Cherenkov radiators (T0) and the Zero Degree Calorimeter (ZDC) [18, 19]. The ZDC detector consists of two sets of calorimeters located at opposite sides with respect to the IP at 116 meters. Each set of detectors consists of two hadronic quartz-fibre "spaghetti" calorimeters, one for the detection of spectator neutrons and the other for the detection of spectator protons. ALICE plans to use initially calculated cross sections and, when available, the TOTEM measurements for luminosity normalization during the proton-proton running phase. Mutual electromagnetic dissociation is considered for normalization during the Heavy Ion phase.

\section{Conclusion}

Luminosity measurements at hadron-lepton and hadronhadron colliders are very challenging. The luminosity uncertainty achieved at HERA I was approximately $1 \%$ and at HERA II (2-3) \% so far. The same uncertainty at the Tevatron is approximately $6 \%$ dominated by the uncertainty in the measurement of the proton-antiproton inelastic cross section. We expect that the lessons learned from HERA and the Tevatron will be very useful for LHC. The expected luminosity uncertainty at the LHC is of the order of $20 \%$ in the beginning and below $5 \%$ after a few years of experience.

I would like to thank the organizers for a very stimulating Conference. I would also like to thank several colleagues from the Tevatron, HERA and LHC for discussions on the information presented here: B. Casey, Y. Enari, J. Konigsberg, G. Snow, A. Sukhanov and A. Valishev from the Tevatron; V. Boudry, S. Levonian, U. Schneekloth and A. Specka from HERA; H. Burkhardt, P. Grafstrom, V. Halyo, D. Marlow, T. Nayak and O. Schneider from the LHC.

\section{References}

[1] S. Klimenko, J. Konigsberg, T. M. Liss, Fermilab-FN-0741 (2003) and references therein.

[2] D. Acosta et al., Nucl. Instr. and Meth. A 494 (2002) 57.

[3] V. M. Abazov et al., Nucl. Instr. and Meth. A 565 (2006) 463.

[4] T. Andeen et al., Fermilab-TM-2365 (2007).

[5] H. Bethe and W. Heitler, Proc. Roy. Soc., A 146 (1934) 83.

[6] H1 Collaboration, DESY PRC 98/05.

[7] M. Derrick et al., Z. Phys. C 63 (1994) 391; J. Andruszkow et al., Acta Phys. Pol. B 32 (2001) 2025.

[8] M. Helbich et al., Nucl. Instr. and Meth. A 565 (2006) 572.

[9] H1 Collaboration, paper pa17-026, submitted to the XXVIII ICHEP at Warsaw (1996); http://www-h1.desy.de/h1det/lumi/.

[10] E. Bravin et al., PAC’07 proceedings, FRPMN067.

[11] H. Burkhardt, P. Grafstrom, LHC Project Report 1019, (2007).

[12] ATLAS Collaboration, Technical Design Report, CERN/LHCC 99-14.

[13] CMS Collaboration, Technical Design Report, CERN/LHCC 97-31.

[14] CDF Collaboration, A. Abulencia et al., Phys. Rev. Lett. 98112001 (2007).

[15] ATLAS Collaboration, Letter of Intent, CERN/LHCC 2004-010; LHCC I-014.

[16] TOTEM Collaboration, Technical Design Report, CERN/LHCC 200402.

[17] M. Ferro-Luzzi, Nucl. Instr. and Meth. A 553 (2005) 388.

[18] ALICE Collaboration, Technical Design Report, CERN/LHCC 2004025.

[19] ALICE Collaboration, Technical Design Report, CERN/LHCC 99-5. 